\title{
Evaluation of Bone Turnover in Patients With Type 1 Diabetes Mellitus
}

\author{
Sain Safarova
}

\begin{abstract}
Background: The purpose of the study was to assess the effect of changes in male and female's body with type 1 diabetes mellitus (T1DM) on the state of the bone mineral density and the metabolic rate, and to determinate the direction of changes in serum markers of bone remodeling and bone mineral density in both sexes due to this disease.

Methods: Bone mineral density (with dual-energy X-ray absorptiometry (DXA)) and serum bone remodeling markers (alkaline phosphatase (ALP), procollagen type 1 intact N-terminal propeptide (P1NP) and C-terminal cross-linking telopeptide of type I collagen (b-CTx)) were measured in 57 female and 41 male with T1DM $(n=98)$ and in a group control, consisting of 43 female and 39 male $(n=82)$.
\end{abstract}

Results: Analysis of the obtained results showed lower values in all the surveyed areas of patients with diabetes, in comparison with the control group. It was found that the studied bone formation markers (ALP, P1NP) behave differently. While the P1NP level is significantly reduced in female with T1DM vs. control group patients $(\mathrm{P}<0.001)$, the level of ALP was comparable between the groups of patients with T1DM and control $(\mathrm{P}>0.05)$. B-CTx showed a significant negative correlation with the $\mathrm{T}$-score of lumbar spine $(\mathrm{r}=-0.431, \mathrm{P}=0.000)$.

Conclusions: Based on the results of studying the T-score it was found that in male and female with diabetes, in comparison with the control group, the bone density in the vertebrae was reduced. In men with T1DM changes in the T-score in the spine were found less intense than those in female.

Keywords: Diabetes mellitus type 1; Bone mineral density; Osteoporosis

\section{Introduction}

Diabetes mellitus (DM) is a chronic metabolic disorder with common late complications, which included diabetes-associat-

Manuscript submitted January 3, 2018, accepted January 18, 2018

Department of Internal Diseases III, Azerbaijan Medical University, Baku, Azerbaijan. Email: sainsafarova@gmail.com

doi: https://doi.org/10.14740/jem483w ed bone alterations that are characterized by a decrease in bone quality, leading to an increased risk of bone fracture. Diabetic osteopathy includes: osteopenia, osteoporosis, Sharko arthropathy and diabetic foot syndrome [1,2].

The mechanisms of DM induced bone fragility are complex and only partially understood. It was thought that various disorder mechanism of calcium metabolism are described, such as a disorder of calcium absorption in the intestine, an increased calcium loss in the urine, followed by the development of secondary hyperparathyroidism and bone loss [3].

Bone metabolism in diabetes is characterized by a decrease in the function of osteoblasts, in connection with which, a disorder of bone formation is not able to compensate for normal or increased bone resorption. Disturbance of metabolic processes in patients bone tissue with DM can be caused by the direct effect of insulin deficiency and/or hyperglycemia on bone and bone marrow, by the end products of glycation of bone matrix proteins that disrupt the synthesis of bone collagen, the production of inflammatory cytokines, adipokines and their damaging effect on cells of bone and disorder of neuromuscular regulation $[4,5]$.

Additional factors for diabetic osteopenia may be an abnormality of blood circulation in the bone with micro- and macroangiopathy and a decrease in the mechanical stress on the bone associated with polyneuropathy and myopathy [6]. Another cause of diabetic osteopenia in type 1 diabetes mellitus (T1DM) is the accumulation of low peak bone mass during puberty, in part due to osteoblast dysfunction, partly related to the need for diet and physical activity.

Whether considering changes in bone tissue with an increased risk of a fracture in DM associated directly with metabolic changes occurring in this disease is a discussion subject. Some studies suggest that in patients with T1DM, the risk of fracture of the vertebrae and femoral neck increases $[7,8]$.

The purpose of the study was to assess the effect of changes in male and female's body with T1DM on the state of bone mineral density and its metabolic rate, and to determinate the directionality of changes in serum markers of bone remodeling and bone mineral density in both sexes due to this disease.

\section{Materials and Methods}

A cross-sectional study was carried out in 98 patients with T1DM (female (F) 57: male (M) 41), who had not been previously diagnosed with bone metabolism disorders and osteoporosis. Patients with diabetes were 40 to 68 years old (55.89 
Table 1. Characteristics of Subjects

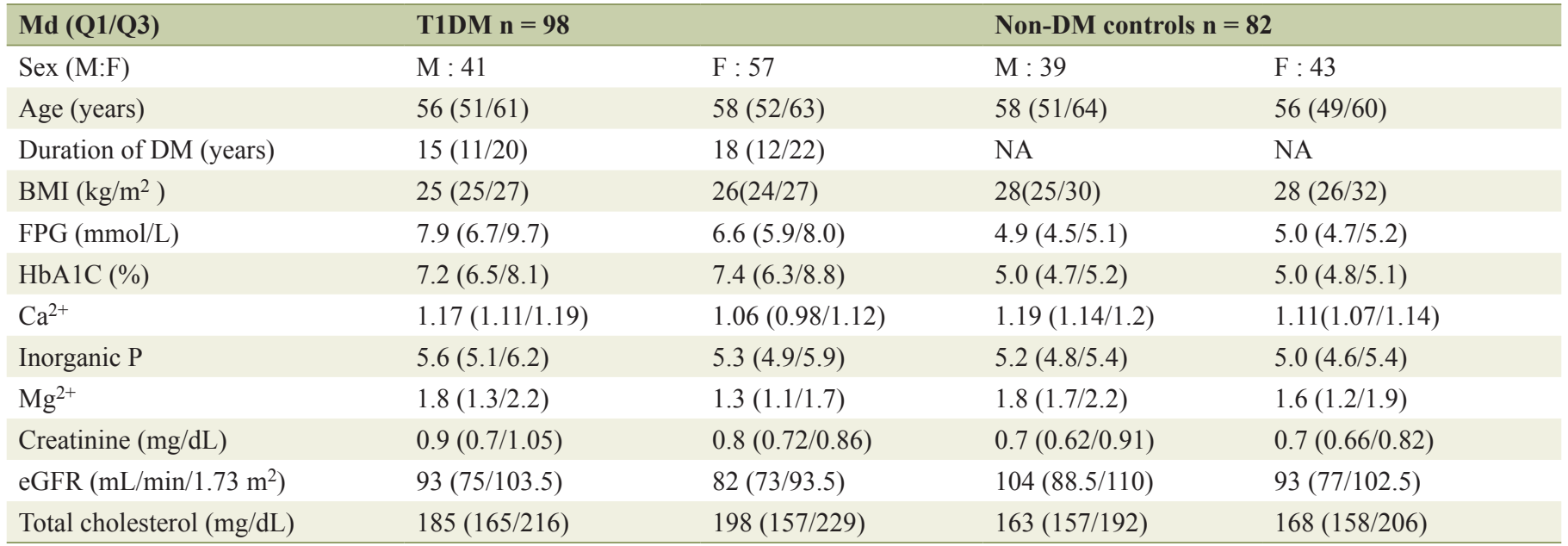

M: male; F: female.

\pm 0.7 years, mean $\pm \mathrm{SD}$ ). The duration of diabetes was 16.6 \pm 0.6 years. The control group consisted of 82 patients (F: 48 and $\mathrm{M}: 34,55.97 \pm 0.9$ years, mean \pm SD) without a history of diabetes.

Patients with past history of fracture, steroid abuse, renal failure, liver failure, malignant disease, rheumatoid arthritis, thyroid and parathyroid disease and other endocrinopathies were excluded from the study. Each subject underwent measurement of hip and spine (L1-L4) bone mineral density (BMD) using dual-energy X-ray absorptiometry (DXA, Hologic Discovery QDR-4500A, USA).

Bone metabolism were examined by measuring serum alkaline phosphatase (ALP), procollagen type 1 intact $\mathrm{N}$-terminal propeptide (P1NP) as markers of bone formation and serum $\mathrm{C}$ terminal cross-linking telopeptide of type I collagen (b-CTX) as marker of bone resorption. P1NP and b-CTX measurement was taken by the electrochemiluminescence immunoassay (ECLIA) method using the Cobas e411 (Roche Diagnostics, Switzerland). Other collected data include levels of $\mathrm{HbAlC}, \mathrm{Ca}^{2+}$, inorganic $\mathrm{P}^{+}, \mathrm{Mg}^{2+}$, creatinine, eGFR and total cholesterol.

Statistical analysis was carried out using BioStat Pro 6.2.2.0 program. Data were presented as median (Md) and interquartile range $(\mathrm{Q} 1 / \mathrm{Q} 3)$ or number $(\%)$ unless specified otherwise. Statistical analysis was done using unpaired parametric data analyzed by Mann-Whitney U test. Spearman's rank correlation was calculated to assess the power of connection between the parameters. $\mathrm{P}<0.05$ was considered statistically significant.

\section{Results}

Characteristics of subjects and clinical characteristics for the study groups are shown in Table 1.

The analysis of the obtained results showed lower values in all the surveyed areas of patients with diabetes, in comparison with the control group. Statistically in the L1-L4 region, BMD T-score in female was significantly lower than that in the male's group with T1DM ( $\mathrm{P}<0.005)$, as in female, in $72 \%$ cases, and in male already in $54 \%$ of cases. According to the average T-score, it was found that in both male and female with diabetes, bone density in the vertebrae was reduced. BMD Tscore in patients with T1DM in the femoral neck region was significantly lower in both male and female approximately in the same percentage of cases than in the control group, but still more often in male $(41 \%)$. The results of BMD characteristics and bone metabolism markers in the diabetic and control groups are presented in Table 2.

As shown, in male with T1DM, changes in the lumbar spine T-score were found being lower intensity than in female $(\mathrm{P}<0.005)$. A similar situation was observed in the femoral neck area, where divergences in bone density in male and female with T1DM also differ significantly $(\mathrm{P}<0.05)$.

At T1DM, a negative, statistically significant correlation was found between the T-score of the proximal femur and the duration of diabetes in the male group: $\mathrm{r}=-0.510, \mathrm{P}=0.01$. The negative correlation was also noted in the female group: $\mathrm{r}=-0.568, \mathrm{P}=0.001$. Also, a negative, statistically significant correlation was found between the change in T-score measured in the femoral neck region and the duration of diabetes in the female group: $r=-0.460, P=0.01$.

Statistically in male with T1DM, significantly lower Zscore values was observed in femoral neck and lumbar spine areas in comparison with the control group $(\mathrm{P}<0.05$ and $\mathrm{P}<$ $0.001)$. At the same time, the results of the comparative analysis of BMD (Z-score) in male and female with T1DM in axial skeleton testify to the absence of significant differences in the degree of bone loss $(\mathrm{P}>0.05)$. The $\mathrm{Z}$-score in male with $\mathrm{T} 1 \mathrm{DM}$ in the proximal femur was not significantly different from the control group $(\mathrm{P}>0.05)$.

These data showed that there was a statistically significant decrease in the BMD of the lumbar spine, femoral neck and proximal femur in female with DM in comparison with the control group. The difference was expressed for the BMD of the lumbar spine and femoral neck, where a higher percentage of patients had a lower bone mass compared to the BMD of the 
Table 2. Bone Turnover Markers and BMD Characteristics of Subjects

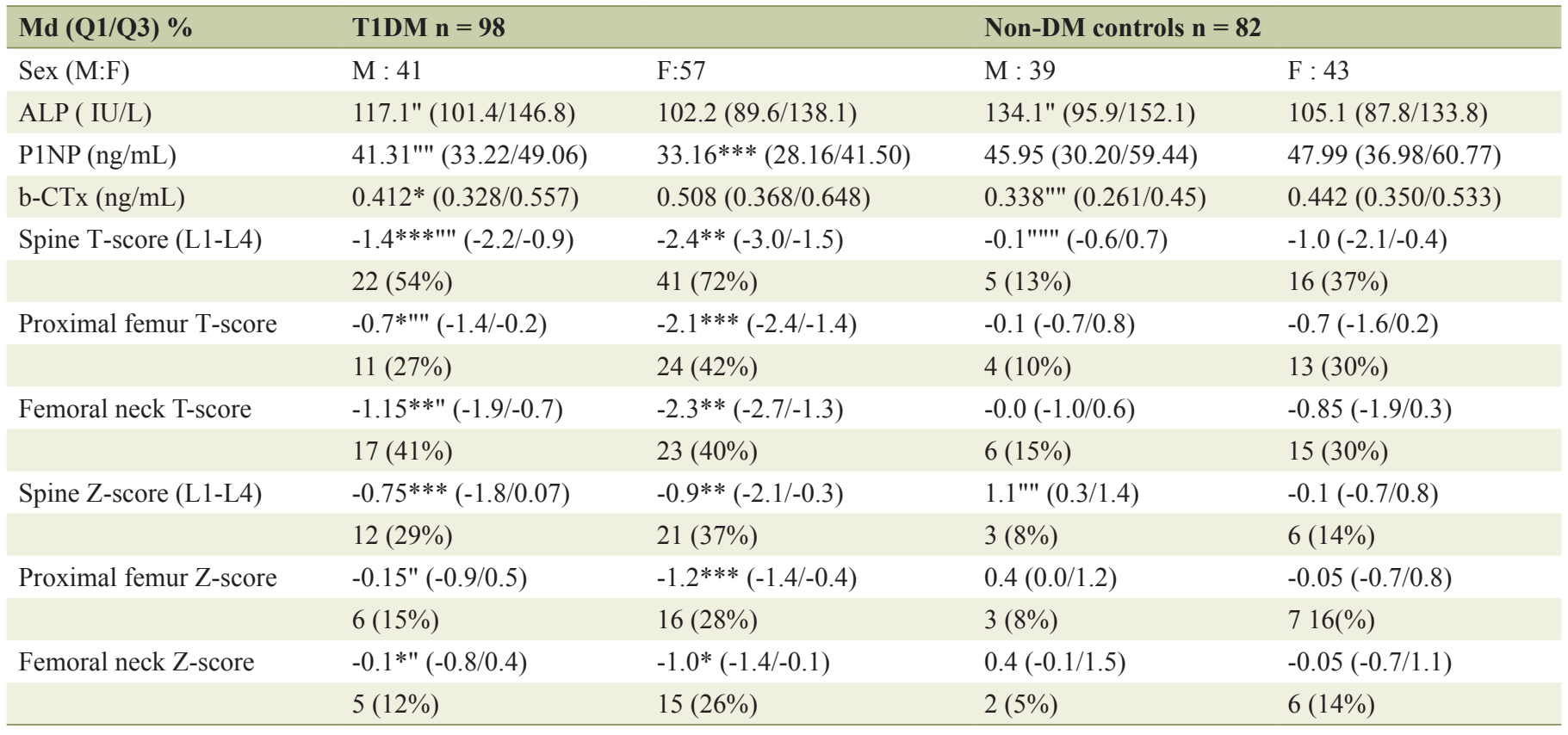

${ }^{*} \mathrm{P}<0.05,{ }^{* *} \mathrm{P}<0.005,{ }^{* * *} \mathrm{P}<0.001$ compared with patients of the control group; "P $<0.05$, "'"P $<0.005$, "'"'P $<0.001$ compared with female patients of the same group.

proximal femur.

In male with T1DM, the mineral density of L1-L4 was also lower in comparison with the control group. We found a statistically significant lower BMD of the femoral neck and lumbar spine in male with diabetes in comparison with the control group. The difference was statistically significant, as in the female group $(\mathrm{P}<0.005$ and $\mathrm{P}<0.001)$.

There was a significant correlation of BMD measured at two different sites of the skeleton, indicating that for general practice, BMD evaluation in patients with T1DM can be performed at any site, but changes in the lumbar spine are more pronounced.

Also, the prevalence of left-right-sided differences in the mineral density of the hips (T-score BMD) by DXA and the frequency of the lowest BMD values was determined in the study while comparing the L1-L4 section of the lumbar spine shown in Table 3.

Thus, BMD in L1-L2 lumbar vertebrae was the lowest in seven $(17 \%)$ cases among the male surveyed and in $10(17.5 \%)$ female; in L3-L4 lumbar vertebrae in $15(36.5 \%)$ and $31(54 \%)$ of cases in the group of patients with T1DM. In this case, L4 had the lowest BMD. In the proximal femur, the following changes were observed in the T-score BMD: left hip in 10 $(24 \%)$ males and $13(23 \%)$ females, and on the right in seven $(17 \%)$ males and $10(17.5 \%)$ females of T1DM patients. The lowest T-score was found in female in the lumbar spine and in the left hip, accounting for a total of $42 \%$ and $13 \%$ of the total number of T1DM patients.

It was found that the studied bone formation markers (ALP, P1NP) behave differently; while the P1NP level was significantly reduced in female with T1DM in comparison with control group patients $(\mathrm{P}<0.001)$, the ALP level was comparable between the groups of patients with T1DM and control group $(\mathrm{P}>0.05)$.

Also, there was a positive correlation between ALP and T1DM duration $(r=0.200, P=0.04)$. B-CTx showed a significant negative correlation with the T-score of the BMD of the lumbar spine $(\mathrm{r}=-0.431, \mathrm{P}=0.000)$. A significant correlation was found between the level of $\mathrm{HbAlc}$ in the serum with the P1NP bone formation marker in the T1DM group, with a negative Spearman correlation coefficient $(r=-0.252, P=0.03)$. In addition, negative correlation of P1NP with T1DM duration was observed $(r=-0.210, P=0.03)$. Significant positive correlations were observed between the b-CTx and duration of $\operatorname{T1DM}(\mathrm{r}=0.273, \mathrm{P}=0.007), \operatorname{ALP}(\mathrm{r}=0.213, \mathrm{P}=0.03)$.

\section{Discussion}

The data showed that the observed low bone mineral density

Table3. A Comparison of the Distribution of T-score BMD Reduction According to WHO Classification Between the Lowest Indices of Both Hips and Spine at Patients With T1DM and Control Group

\begin{tabular}{lllll}
\hline$\%$ & \multicolumn{2}{l}{ T1DM $\mathbf{n}=\mathbf{9 8}$} & \multicolumn{2}{l}{ Non-DM controls n=82 } \\
\hline Sex (M : F) & M : 41 & F : 57 & M : 39 & F : 43 \\
L1 - L2 & $7(17 \%)$ & $10(17.5 \%)$ & $1(2.5 \%)$ & $5(12 \%)$ \\
L3 - L4 & $15(36.5 \%)$ & $31(54 \%)$ & $4(10 \%)$ & $11(25.5 \%)$ \\
Left hip & $10(24 \%)$ & $13(23 \%)$ & $5(13 \%)$ & $9(21 \%)$ \\
Right hip & $7(17 \%)$ & $10(17.5 \%)$ & $1(2.5 \%)$ & $6(14 \%)$ \\
\hline
\end{tabular}


in patients with diabetes is associated with an increased bone resorption. The level of bone resorption marker b-CTx in patients with diabetes was higher in comparison with control group. Thus, in male with T1DM, a statistically significant increase in the level of b-CTx $(\mathrm{P}<0.05)$ was observed in comparison with the control group, which indicates an increased bone resorption in this group. Similar results were obtained in number of other authors' studies $[4,6]$.

The propensity to low-traumatic fractures in diabetes is associated with changes in the quality of the organic component of bone tissue, under the influence of glycosylation processes of proteins acting on the metabolism of bone tissue and its strength, changing the processes of osteoblastogenesis and osteoclastogenesis $[9,10]$.

A number of studies have also demonstrated a divergence in the processes of bone formation in diabetes $[4,6]$. One possible explanation for this discrepancy may be that different markers of bone formation reflect different stages of osteoblast differentiation. While ALP is produced by mature osteoblasts, P1NP is formed during the phase of procollagen synthesis [3]. These studies also confirm the more pronounced decrease in P1NP in patients with T1DM compared with control group $[6,7]$.

\section{Conclusions}

Taking into account data from studies showing an increased risk of fractures in patients with T1DM, this disease can be considered an important risk factor for secondary osteoporosis. BMD can be studied at both sites of the axial skeleton due to a significant correlation of osteodensitometric results. Based on the results of studying the T-score, it was found that in both male and female with diabetes, in comparison with the control group, the bone density in the vertebrae was reduced. In male with T1DM, changes in the T-score in the spine were found less intense than those in female. The level of b-CTx showed a statistically significant negative correlation with the BMD of the lumbar spine, consisting mainly of a spongy bone with high metabolic activity. This indicates that both bones metabolism markers and DXA can be considered as independent indicators of changes in bone tissue, which can be of great importance for early diagnosis and evaluation of the effectiveness of the therapy.

\section{Conflict of Interest}

None identified.

\section{References}

1. Liao CC, Lin CS, Shih CC, Yeh CC, Chang YC, Lee YW, Chen TL. Increased risk of fracture and postfracture adverse events in patients with diabetes: two nationwide population-based retrospective cohort studies. Diabetes Care. 2014;37(8):2246-2252.

2. Jackuliak P, Payer J. Osteoporosis, fractures, and diabetes. Int J Endocrinol. 2014;2014:820615.

3. AlzubaidiSA., Bin Salman AI, Alguraigari AA, et al. Systematic review of bone turnover Biochemical markers in diabetes mellitus (DM). International Journal of Healthcare Sciences. 2016;4:41-48.

4. Farlay D, Armas LA, Gineyts E, Akhter MP, Recker RR, Boivin G. Nonenzymatic Glycation and degree of mineralization are higher in bone from fractured patients with type 1 diabetes Mellitus. J Bone Miner Res. 2016;31(1):190-195.

5. Rubin MR. Bone cells and bone turnover in diabetes mellitus. Curr Osteoporos Rep. 2015;13(3):186-191.

6. Hough FS, Pierroz DD, Cooper C, Ferrari SL, Bone IC, Diabetes Working G. MECHANISMS IN ENDOCRINOLOGY: Mechanisms and evaluation of bone fragility in type 1 diabetes mellitus. Eur J Endocrinol. 2016;174(4):R127-138.

7. Alexopoulou O, Jamart J, Devogelaer JP, Brichard S, de Nayer P, Buysschaert M. Bone density and markers of bone remodeling in type 1 male diabetic patients. Diabetes Metab. 2006;32(5 Pt 1):453-458.

8. Pramojanee SN, Phimphilai M, Chattipakorn N, Chattipakorn SC. Possible roles of insulin signaling in osteoblasts. Endocr Res. 2014;39(4):144-151.

9. Al-Zaabi K, Badr HE, Mahussain S, Mohammad M, AlNafisi N. Bone mass density in diabetic women: is there a detrimental effect? Middle East J Age Aging. 2008;5:1217.

10. Rubin MR. Skeletal fragility in diabetes. Ann N Y Acad Sci. 2017;1402(1):18-30. 